\title{
The MicroBooNE Experiment
}

\section{Benjamin J.P. Jones ${ }^{1}$}

Massachusetts Institute of Technology

77 Massachusetts Avenue, Cambridge, MA, United States of America

E-mail: bjpjones@mit.edu

The high resolution 3D tracking offered by liquid argon time projection chambers make them an excellent candidate for future neutrino detectors in the next generation of long baseline experiments. In order to utilize this emerging detector design for such experiments, the scalability and physics capabilities of the technology must be proven. The MicroBooNE experiment is a 170 ton liquid argon time projection chamber detector which will run in the booster neutrino beam at Fermilab, starting in 2014. The experiment incorporates two sensitive subsystems: a time projection chamber with a $2.5 \mathrm{~m}$ drift, which provides high resolution $3 \mathrm{D}$ tracking by measuring ionization charge deposits from charged particles traversing the argon bulk; and an optical system comprised of cryogenic photomultiplier tubes, which will measure scintillation light and provide a trigger and few-nanosecond level timing information.

The 2011 Europhysics Conference on High Energy Physics-HEP 2011,

July 21-27, 2011

Grenoble, Rhône-Alpes France

\footnotetext{
${ }^{1}$ Speaker
} 


\section{Physics Goals}

MicroBooNE is a 170 Liquid Argon Time Projection Chamber (LArTPC) which will begin taking data in the Booster Neutrino Beam at Fermilab in early 2014. The detector will have excellent particle ID capabilities for low energy tracks produced in neutrino interactions. As well as providing a training ground for US LArTPC technology ahead of a possible giant liquid argon detector for the LBNE experiment, the physics goals of the experiment fall broadly into three categories:

\subsection{Resolution of the MiniBooNE Anomalies}

The MiniBooNE experiment ran at a baseline of $\sim 500 \mathrm{~m}$ in a muon neutrino beam of peak energy $1 \mathrm{GeV}$, with the goal of confirming or refuting an anomalous short baseline neutrino oscillation signal reported by the LSND collaboration [1]. Anomalous electron neutrino appearance was observed at low energy in both neutrino and antineutrino modes, and an intermediate "LSND-like" excess was observed in antineutrino mode only [2]. MicroBooNE will investigate both LSND-like and low energy excesses by utilizing the same beam at approximately the same baseline as MiniBooNE but with a different detection technology. Unlike in a Cerenkov detector, the high resolution tracking and calorimetry of an LArTPC allows the separation of electrons and gammas as the primary particles in electromagnetic showers, which could rule out photon production in neutrino scattering as an explanation of the low energy events.

\subsection{Low Energy Neutrino-Argon Cross Sections}

The current MicroBooNE run plan will result in the collection of $\sim 130,000 v$-Ar interaction events in a $1 \mathrm{GeV}$ beam. The excellent energy resolution and PID capabilities of a LArTPC detector, especially at low energies, means a wide range of cross section measurements are uniquely accessible.

\subsection{Selected Nonaccelerator Physics Topics}

MicroBooNE is capable of observing neutrinos produced by a burst supernova in our galaxy. It will also help constrain proton decay backgrounds for future giant detectors, with measurements of both neutrino induced and cosmogenic kaon production.

\section{The MicroBooNE Detector Subsystems}

\subsection{Time Projection Chamber}

Charge deposited in the 70 ton fiducial volume is drifted under the influence of a $500 \mathrm{~V} / \mathrm{cm}$ electric field, towards three crossed wire planes at the edge of the detector, each with a $3 \mathrm{~mm}$ wire pitch. The wire pitch directions are vertical and at \pm 60 degrees. The chamber will be strung with 8256 wires beginning this summer at Yale and Syracuse universities. Final assembly of the TPC and insertion into the cryostat (Figure 1, Left) will then take place in the D0 hall at Fermilab in 2012-2013.

\subsection{Optical System}

The MicroBooNE optical system consists of 30 cryogenic PMTs mounted behind wavelength shifting plates, which record the $128 \mathrm{~nm}$ scintillation light produced by charged particles 
traversing the liquid argon active volume. The PMT units for MicroBooNE, each incorporating one PMT, wavelength shifting plate, bespoke cryogenic base, combined signal / HV cable and PEEK plastic frame will be assembled, tested and characterized in a cryogenic test stand at Fermilab this autumn. Simulations have shown the system's ability to act as an effective trigger for the design benchmark of $40 \mathrm{MeV}$ proton tracks, and suggest high trigger efficiency for tracks with as little as $5 \mathrm{MeV}$ of energy deposition. Reconstruction applications using the measured scintillation light are also under investigation, including pulse shape discrimination techniques and the application of corrections for charge losses during drift.

\subsection{Active Volume and Cryogenics}

In order to maintain argon purity, temperature and pressure uniformity, MicroBooNE employs an extensive system of cryogenics. Constant argon purification is employed to combat outgassing from the cryostat, with electron lifetime based purity monitors both inside the detector and in the argon circulation system. Temperature is maintained by an LN2 refrigeration system. Electronegative impurities in liquid argon can absorb free charges during the drift to the wire planes, so dissolved oxygen and water levels must be maintained at less than 100ppt. Other impurities also quench scintillation light production, and so nitrogen must also be maintained at less than $2 \mathrm{ppm}$. Temperature inhomogeneities can lead to non-uniformity of drift velocity and hence image distortion, and simulations show that the temperature uniformity can be comfortably ensured within $0.1 \mathrm{~K}$ across the volume.

\subsection{Software and Analysis Tools}

Simulation, reconstruction and data analysis jobs for MicroBooNE are performed using the LArSoft software package, which is a general-purpose framework for liquid argon TPC experiments. Some LArSoft tools have already been proven in the analysis of data from the ArgoNeuT prototype[3] and advanced methods such as e/gamma separation algorithms (Figure 1, Right) are in a state of continuing development.
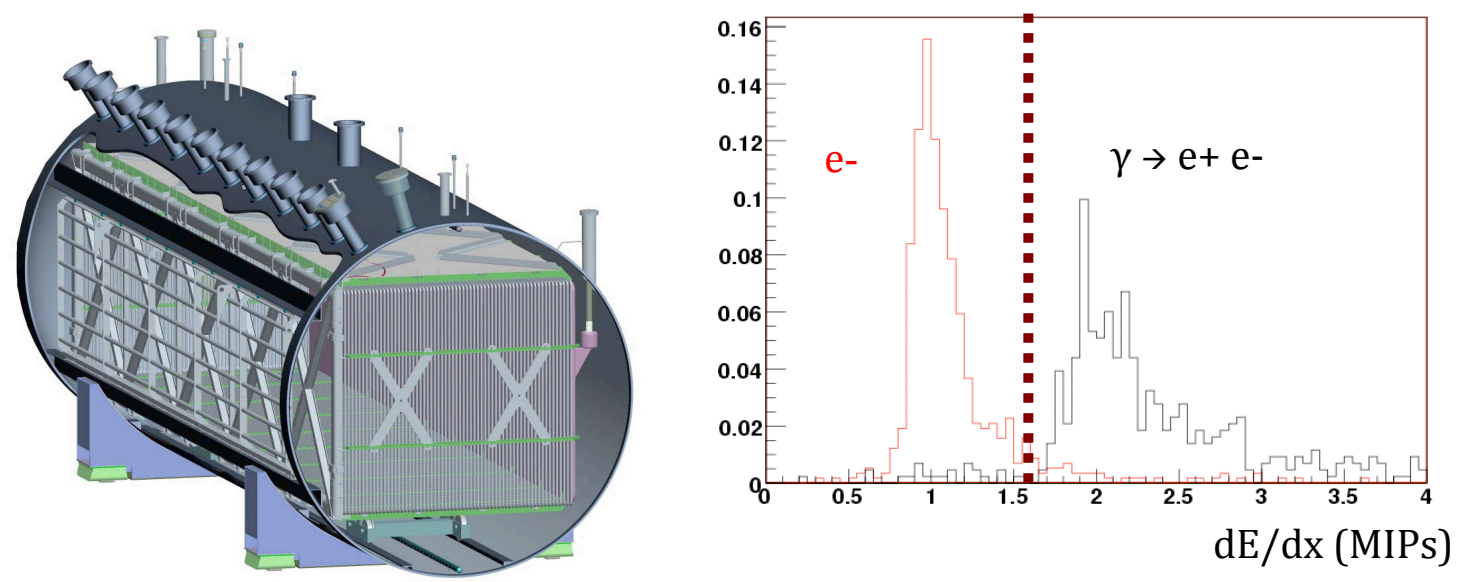

Figure 1: Left: MicroBooNE cryostat cutaway showing TPC and field cage. Right: preliminary e/gamma separation capability based on $\mathrm{dE} / \mathrm{dx}$ in first $24 \mathrm{~mm}$ of shower for $250 \mathrm{MeV}$ electrons vs $250 \mathrm{MeV}$ photons

\section{Conclusion}

MicroBooNE is a 170 ton liquid argon neutrino experiment, comprising of a time projection chamber with 70 ton fiducial volume and a scintillation light collection system. MicroBooNE will observe the interactions of neutrinos from the Booster Neutrino Beam at Fermilab and make a clear statement about the nature of the MiniBooNE low energy excesses and LSND-like anomaly. MicroBooNE will also 
measure low energy $v$-Ar cross sections and backgrounds for future proton decay searches. Construction and testing of the sensitive detectors for MicroBooNE is under way and will continue through 2012, with final assembly of the detector following in 2013.

\section{References}

[1]A. Aguilar et al., Evidence for neutrino oscillations from the observation of $\overline{v_{e}}$ appearance in a $\overline{v_{\mu}}$ beam, Phys. Rev. D64 (2001) 112007

[2]Aguilar-Arevalo A A et al, Unexplained Excess of Electron-Like Events From a 1-GeV Neutrino Beam Phys. Rev. Lett. 102 (101802) [hep-ex/0812.2243], Event Excess in the MiniBooNE Search for $v_{\mu} \rightarrow v_{e}$ Oscillations, Phys. Rev. Lett. 105 (181801) [hep-ex/1007.1150]

[3]C. Anderson et al, First Measurements of Inclusive Muon Neutrino Charged Current Differential Cross Sections on Argon [hep-ex/1111.0103] 